\title{
Anisotropic adaptive resolution of boundary layers for heat conduction problems
}

\author{
M. Breuß, V.Dolejšś, A. Meister
}

April 6, 2005

\begin{abstract}
We deal with the numerical solution of heat conduction problems featuring steep gradients. In order to solve the associated partial differential equation a finite volume technique is used and unstructured grids are employed. A discrete maximum principle for triangulations of a Delaunay type is developed. To capture thin boundary layers incorporating steep gradients an anisotropic mesh adaptation technique is implemented. Computational tests are performed for an academic problem where the exact solution is known as well as for a real world problem of a computer simulation of the thermoregulation of premature infants.
\end{abstract}

Keywords: Finite volume method, Thermoregulation, Maximum principle, Anisotropic adaptation

\section{Introduction}

The numerical simulation of flow phenomena often incorporates the evaluation of boundary layers or, more generally, of physical states which involve steep gradients of the occuring quantities, see $[19,20,13]$. This can be done either by the use of a sensible analytical approximation, e.g. by employing the Boussinesq approximation in a suitable situation [15], or by using a very fine grid near the boundary to achieve a valid numerical approximation of the flow field within the boundary layer.

The latter strategy can be advantageous since the computed solution does not rely on additional physical assumptions. Furthermore, in some situations there is no analytical approximation available and the flow in the boundary layer has to be computed numerically anyway, e.g. in the case of heat equations with complicated source terms on a sophisticated domain featuring different boundary conditions. However, if the numerical simulation of the flow within a boundary layer is realized via an uniformly fine grid, the resulting computational problem easily becomes very large. This assertion remains true even when an 
adaptive procedure yielding locally uniformly fine grid structures is applied. Clearly, an anisotropic refinement technique offers a sensible approach to deal with such a problem since it allows to use anisotropic grid structures within a boundary layer, i.e., it allows grid structures to be long and thin which fits with the characterization of a boundary layer problem.

Within this paper, we employ the anisotropic mesh adaptation (AMA) technique developed in $[8,9]$ within a finite volume framework. The AMA technique does not depend on the exact formulation of the problem, thus it allows a wide range of applications.

A distinctive feature of the employed finite volume method is given by the special implementation of the boundary conditions. By the concept of extended barycenters introduced in [12], the method is well-defined for general non-convex triangulations. Furthermore, this concept allows to model the temperature directly at the edges of the computational domain. This not only distinguishes our method, the possibility to manipulate the physical fluxes directly at the boundary can be advantageous from the modeling point of view. Exactly this is the case in extensions of the industrial application we consider; e.g. while the relevant temperature on the body surface of a premature infant inside an incubator depends also on the ventilation, the degree of heat exchange between the body and the surroundings depends on the vasomotion of the skin directly adjacent to its surface, see $[5,24,4]$ and the references therein.

The resulting numerical method is shown to offer an effective and useful tool for the computation of sophisticated flow fields with boundary layers in two spatial dimensions over general non-convex domains. In order to demonstrate this, several test cases are considered.

The mathematical problems under consideration are given by variations of the heat equation

$$
\partial_{t} T(\mathbf{x}, t)=\operatorname{div}(\lambda(\mathbf{x}) \nabla T(\mathbf{x}, t))+q(\mathbf{x}, t),
$$

where $\mathbf{x}=\left(x_{1}, x_{2}\right)^{T} \in \Omega \subset \mathbf{R}^{2}$ and $t \in \mathbf{R}^{+}$. Note that the sources may be quite complicated as it is e.g. the case in the industrial application we consider. Additionally, initial and boundary conditions of various types are employed. As indicated before, the spatial domain $\Omega$ may be non-convex and quite sophisticated depending on the concrete application.

This paper is organized as follows. At first, we briefly describe the finite volume scheme recently introduced by Fischer et al. $[12,3]$. Then a discrete formulation of the maximum principle is proven. Thereby, in comparison to [12] weaker assumptions concerning the underlying primary mesh are used which especially motivates the application of an anisotropic refinement procedure. After that, the used anisotropic mesh adaptation technique is introduced. We compare several analytical solutions featuring strong boundary layers with results computed with the help of the described method. Afterwards the method is applied to a real world problem, namely a model of thermoregulation of premature infants. 


\section{The Numerical Method}

Finite volume methods have proven to be reliable and accurate approximation techniques $[2,19,20,22,17,12,10,11]$. These schemes are formulated on general control volumes and deal with the weak formulation of the underlying problem. The function $T$ is called a weak solution of equation (1), if

$$
\left.\frac{d}{d t}(\mathcal{M} T)(t)\right|_{\sigma}=-\frac{1}{|\sigma|} \int_{\partial \sigma} \lambda(\mathbf{x}) \nabla T(\mathbf{x}, t) \cdot \mathbf{n} d s+\frac{1}{|\sigma|} \int_{\sigma} q(\mathbf{x}, t) d \mathbf{x}
$$

holds for all control volumes $\sigma \subset \Omega$, where $\mathcal{M}$ is the cell average operator, and $|\sigma|$ and $\mathbf{n}$ denote the area of the box $\sigma$ and the unit outer normal vector on $\partial \sigma$, respectively.

In order to obtain an appropriate decomposition of the domain $\Omega$ into a finite number of control volumes, we start from an arbitrary conforming triangulation $\mathcal{D}_{h}$ of the domain $\Omega$ which is called the primary mesh [22], consisting of finitely many triangles $\mathcal{D}_{i}, i \in I=$ $\left\{1, \ldots, \# \mathcal{D}_{h}\right\}$. Furthermore, $\mathcal{N}_{h}$ denotes the index set of all nodes of the triangulation $\mathcal{D}_{h}$ and is subdivided by $\mathcal{N}_{h}=\mathcal{N}_{h}^{\mathcal{D}_{h}} \cup \mathcal{N}_{h}^{\partial \mathcal{D}_{h}}$, where $\mathcal{N}_{h}^{\mathcal{D}_{h}}$ is associated with the inner points and $\mathcal{N}_{h}^{\partial \mathcal{D}_{h}}$ includes the indices of the boundary points. We set $N:=\# \mathcal{N}_{h}$ and denote the three edges of the triangle $\mathcal{D}$ by $e_{\mathcal{D}, k}, k=1,2,3$. Furthermore, we define

$$
\begin{gathered}
E(i)=\left\{e_{\mathcal{D}, k} \mid k \in\{1,2,3\}, \mathcal{D} \in \mathcal{D}_{h}, \text { node } \mathbf{x}_{i} \in e_{\mathcal{D}, k}\right\}, \\
V(i)=\left\{\mathcal{D} \mid \text { node } \mathbf{x}_{i} \text { is vertex of } \mathcal{D} \in \mathcal{D}_{h}\right\},
\end{gathered}
$$

and

$$
C(\mathcal{D})=\left\{i \mid i \in\{1, \ldots, N\}, \text { node } \mathbf{x}_{i} \text { is vertex of } \mathcal{D}\right\} .
$$

Concerning the weak formulation (2) of the governing equation (1), the occurrence of second order derivatives within the partial differential equation (1) requires the evaluation of first order derivatives on the boundary of each control volume. Due to this fact we employ a box type method where the computation of a first order derivative on the boundary of each box is straightforward. For the numerical approximation of the involved integrals we follow the proceeding described in $[12,3]$ which we now briefly review.

We define a discrete control volume $\sigma_{i}$ as the open subset of $\mathbf{R}^{2}$ including the node $\mathbf{x}_{i}=$ $\left(x_{i 1}, x_{i 2}\right)^{T}$ and bounded by the straight lines defined by the connection of the midpoint of the edge $e_{\mathcal{D}, k} \in E(i)$ with the barycentre $\mathbf{x}_{s}=\left(x_{s 1}, x_{s 2}\right)^{T}=\frac{1}{3} \sum_{i \in C(\mathcal{D})} \mathbf{x}_{i}$ of the corresponding triangle $\mathcal{D}$. In the case that $\mathbf{x}_{i}$ is on the (piecewise polygonal) boundary of the computational domain, the line defined by the connection of the midpoint of the boundary edge and the node itself is also a part of $\partial \sigma_{i}$. The union $\mathcal{B}_{h}$ of all boxes $\sigma_{i}, i \in \mathcal{N}_{h}$, is called the secondary mesh. Let $N(i)$ denote the index set of all nodes neighboring node $\mathbf{x}_{i}$, i.e., those nodes $\mathbf{x}_{j}$, $j \neq i$, for which $\int_{\partial \sigma_{i} \cap \partial \sigma_{j}} d s \neq 0$ is valid. In general, for $j \in N(i)$ the boundary between the control volume $\sigma_{i}$ and $\sigma_{j}$ consists of two line segments which are denoted by $l_{i j}^{k}, k=1,2$. Furthermore, $\mathbf{n}_{i j}^{k}, k=1,2$, represent the accompanying unit normal vectors.

With regard to the secondary mesh $\mathcal{B}_{h}$ we can rewrite the integral form of the heat equation 


$$
\begin{aligned}
\frac{d}{d t} \underbrace{\left.(\mathcal{M} T)(t)\right|_{\sigma_{i}}=}_{=: T_{i}(t)} & -\frac{1}{\left|\sigma_{i}\right|} \sum_{j \in N(i)} \sum_{k=1}^{2} \int_{l_{i j}^{k}} \lambda(\mathbf{x}) \nabla T(\mathbf{x}, t) \cdot \mathbf{n}(\mathbf{x}) d s \\
& +\frac{1}{\left|\sigma_{i}\right|} \int_{\sigma_{i}} q(\mathbf{x}, t) d \mathbf{x}, \quad \forall \sigma_{i} \in \mathcal{B}_{h} .
\end{aligned}
$$

Taking account of the secondary mesh $\mathcal{B}_{h}$ and using a unique linear distribution of $T$ as well as the mean value of the heat conductivity $\lambda_{\mathcal{D}}$ on each triangle $\mathcal{D}$ of the primary grid directly leads to the formulation of a numerical flux function

$$
H\left(T_{i}(t), T_{j}(t), T_{m}(t) ; \mathbf{n}_{i j}^{k}\right)=\lambda_{\mathcal{D}} \nabla T_{\mathcal{D}}(t) \cdot \mathbf{n}_{i j}^{k} .
$$

Consequently, for each inner control volume we obtain

$$
\int_{\partial \sigma_{i}} \lambda(\mathbf{x}) \nabla T(\mathbf{x}, t) \cdot \mathbf{n}(\mathbf{x}) d s=\sum_{j \in N(i)} \sum_{k=1}^{2} H\left(T_{i}(t), T_{j}(t), T_{m}(t) ; \mathbf{n}_{i j}^{k}\right)\left|l_{i j}^{k}\right| .
$$

A convenient numerical approximation of the remaining boundary integral is required in order to ensure a discrete maximum principle in our numerical framework. Utilizing the notation introduced within Figure 3, Fischer et al. [12] proposed the definition of an extended barycenter

$$
\mathbf{c}_{i}=\mathbf{x}_{i}-\frac{\sqrt{3} \rho_{i}}{4\left\|\mathbf{n}_{i_{1}, \partial \Omega}+\mathbf{n}_{i_{2}, \partial \Omega}\right\|_{2}}\left(\mathbf{n}_{i_{1}, \partial \Omega}+\mathbf{n}_{i_{2}, \partial \Omega}\right),
$$

with a certain weight

$$
\rho_{i}=\frac{1}{2} \min \left\{\left\|\mathbf{x}_{j}-\mathbf{x}_{i}\right\|_{2} \mid j \in N(i) \cap C(\mathcal{D})\right\} .
$$

The consideration of extended barycenters is actually one of the most important aspects of the method. By the consequences of exactly this feature, our method is well-defined even for non-convex computational domains. Furthermore, it enables to model the boundary condition in terms of the temperature at the edges of the computational domain in a very simple way.

The remaining boundary integral is then approximated as

$$
\int_{\partial \sigma_{i} \cap \partial \Omega} \lambda(\mathbf{x}) \nabla T(\mathbf{x}, t) \cdot \mathbf{n}(\mathbf{x}) d s=\sum_{\ell \in\{j, m\}} H\left(T_{i}(t), T_{i, \partial \Omega}\left(\mathbf{x}_{i}\right), T_{i, \partial \Omega}\left(\mathbf{x}_{i \ell}\right) ; \mathbf{n}_{i \ell}\right)\left|l_{i \ell}\right|,
$$

where the physical quantities $T_{i}, T_{i, \partial \Omega}\left(\mathbf{x}_{i}\right)$ and $T_{i, \partial \Omega}\left(\mathbf{x}_{i \ell}\right)$ are assumed to be located at the nodes $\mathbf{c}_{i}, \mathbf{x}_{i}$ and $\mathbf{x}_{i \ell}$, respectively, and the gradient $\nabla T$ is computed using the triangle built by the above mentioned nodes. A simple approximation of the source term yields

$$
\frac{1}{\left|\sigma_{i}\right|} \int_{\sigma_{i}} q(\mathbf{x}, t) d \mathbf{x}=q\left(\mathbf{x}_{i}, t\right) .
$$


For the approximation of the temporal derivative a forward or backward Euler time step technique is employed, whereby the source term is always evaluated in an explicit manner. Thus, the whole numerical method can be written as

$$
\begin{aligned}
T_{i}^{n+1}= & T_{i}^{n}-\frac{\Delta t}{\left|\sigma_{i}\right|}\left\{\sum_{j \in N(i)} \sum_{k=1}^{2} H\left(T_{i}^{m}, T_{j}^{m}, T_{m}^{m} ; \mathbf{n}_{i j}^{k}\right)\left|l_{i j}^{k}\right|\right. \\
& \left.+\delta_{i} \sum_{\ell \in\{j, m\}} H\left(T_{i}^{m}, T_{i, \partial \Omega}\left(\mathbf{x}_{i}\right), T_{i, \partial \Omega}\left(\mathbf{x}_{i \ell}\right) ; \mathbf{n}_{i \ell}\right)\left|l_{i \ell}\right|\right\}+\Delta t q_{i}^{n},
\end{aligned}
$$

where $m \in\{n, n+1\}, \Delta t=t^{n+1}-t^{n}, T_{i}^{n}=T_{i}\left(t^{n}\right), q_{i}^{n}=q\left(\mathbf{x}_{i}, t^{n}\right)$ and

$$
\delta_{i}= \begin{cases}1, & \text { if } i \in \mathcal{N}_{h}^{\partial \mathcal{D}_{h}} \\ 0, & \text { otherwise. }\end{cases}
$$

The consideration of finitely many different tissues within the modelling process of realistic heat conduction problems often yields a heat equation where the heat conductivity is constant almost everywhere. In such a case we usually obtain that the majority of the boxes $\sigma_{i} \in \mathcal{B}_{h}$ satisfies the property $\lambda_{\mathcal{D}}=\lambda_{\widetilde{\mathcal{D}}}$ for all accompanying triangles $\mathcal{D}, \widetilde{\mathcal{D}} \in V(i)$. Examples are given by the academic test case (19) as well as by a realistic model actually employed for the thermoregulation of premarure infants within industrial applications [5]. In this context we are able to improve the maximum principle developed in [12] in the sense that weaker conditions on the underlying primary mesh $\mathcal{D}_{h}$ have to be required. More precisely, angles greater than $\pi / 2$ are now allowed within the triangulation $\mathcal{D}_{h}$. In the following we assume that the primary grid $\mathcal{D}_{h}$ satisfies the properties

- A1: The triangulation $\mathcal{D}_{h}$ is of Delaunay type, whereby the sum of opposite angles $\alpha_{j_{1}}, \alpha_{j_{2}}$ is less than $\pi$ for any pair of neighbouring triangles, see Figure 2,

- A2: The opposite angle $\alpha_{j}$ with respect to a boundary edge is less than $\pi / 2$, see Figure 3 (right).

We now prove the following maximum principle.

Theorem 1 Let $\mathcal{D}_{h}$ satisfy the conditions $\mathrm{A} 1$ and $\mathrm{A} 2$. Given a steady state solution $T_{i}^{n+1}=$ $T_{i}^{n}$ and a vanishing source term $q_{i}^{n}$ for all $i \in\{1, \ldots, N\}$. Given an index $i \in \mathcal{N}_{h}$ such that

$$
T_{i}^{n}=\max _{\ell \in \mathcal{N}_{h}, j \in \mathcal{N}_{h}^{\partial \mathcal{D}_{h}}}\left\{T_{\ell}^{n}, T_{j, \partial \Omega}\right\}
$$

then

$$
T_{\ell}^{n}=T_{i}^{n}=T_{j, \partial \Omega}
$$

holds for all $\ell \in \mathcal{N}_{h}$ and $j \in \mathcal{N}_{h}^{\partial \mathcal{D}_{h}}$.

\section{Proof:}


In the following, we use that both the heat conductivity $\lambda_{\mathcal{D}}$ as well as the gradient of the temperature distribution $\nabla T_{\mathcal{D}}$ are constant on each triangle $\mathcal{D} \in \mathcal{D}_{h}$.

Let an arbitrarily chosen but fixed triangle with a notation as depicted within Figure 1 be given.

By simple geometrical considerations, we can compute the flux over the edges $l_{i j}$ and $l_{i m}$ of the control volume corresponding to the index $i$ as

$$
\begin{aligned}
H & \left(T_{i}, T_{j}, T_{m} ; \mathbf{n}_{i m}\right)\left|l_{i m}\right|+H\left(T_{i}, T_{j}, T_{\ell} ; \mathbf{n}_{i \ell}\right)\left|l_{i \ell}\right| \\
& =-\left(T_{i}-T_{j}\right) \frac{\lambda}{2} \frac{\cos \alpha_{j}}{\sin \alpha_{j}}-\left(T_{i}-T_{m}\right) \frac{\lambda}{2} \frac{\cos \alpha_{m}}{\sin \alpha_{m}} .
\end{aligned}
$$

Note that the identity $\cos \alpha / \sin \alpha=\cot \alpha$ holds, which will be used later on.

Let us first investigate the situation that $i$ and $j$ are not both indices of boundary points, i.e., they belong to two adjacent triangles, see Figure 2.

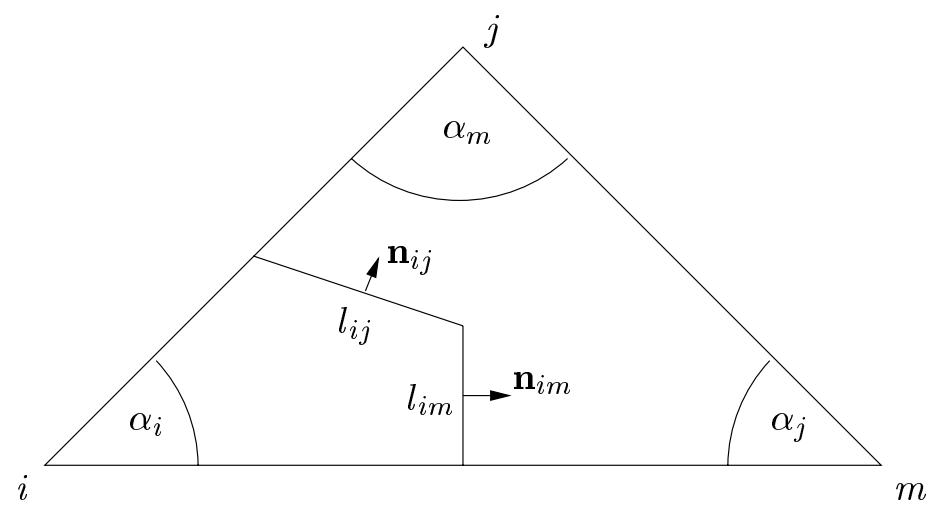

Figure 1: Notations for an arbitrarily chosen triangle.

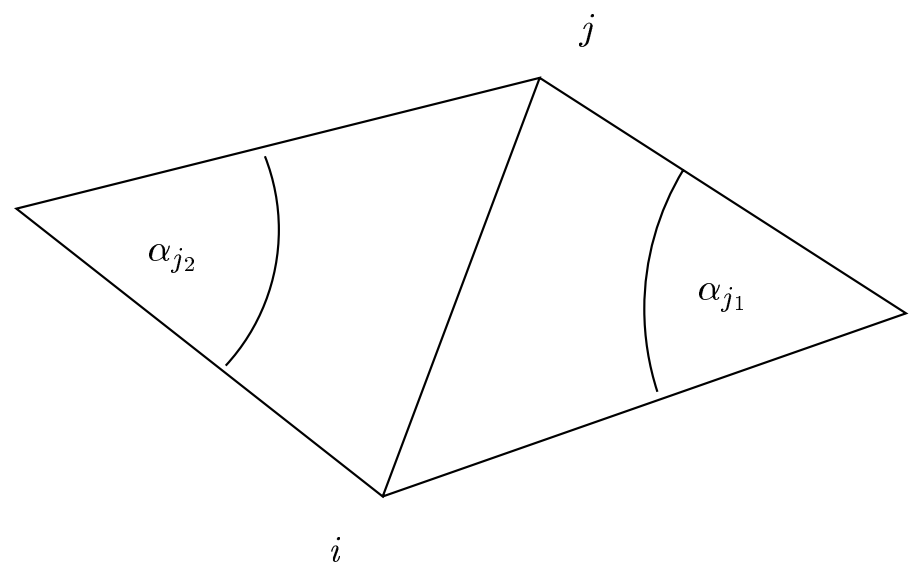

Figure 2: Situation for $i$ and $j$ belonging to two adjacent triangles.

Without restriction on generality, let $i \in \mathcal{N}_{h}^{\mathcal{D}_{h}}$, i.e. $\sigma_{i}$ is an inner control volume and thus 
$\delta_{i}=0$ in (4). We obtain by (4) the equation

$$
0=-\frac{\Delta t}{\left|\sigma_{i}\right|} \sum_{j \in N(i)} \sum_{k=1}^{2} H\left(T_{i}^{m}, T_{j}^{m}, T_{m}^{m} ; \mathbf{n}_{i j}^{k}\right)\left|l_{i j}^{k}\right|=-A_{i i} T_{i}-\sum_{j \in N(i)} A_{i j} T_{j}
$$

with

$$
A_{i j}=-\frac{\lambda \Delta t}{2\left|\sigma_{i}\right|}\left(\cot \alpha_{j_{1}}+\cot \alpha_{j_{2}}\right)=-\frac{\lambda \Delta t}{2\left|\sigma_{i}\right|} \frac{\sin \left(\alpha_{j_{1}}+\alpha_{j_{2}}\right)}{\sin \alpha_{j_{1}} \sin \alpha_{j_{2}}} \stackrel{\mathrm{A} 1}{<} 0
$$

and

$$
A_{i i}=-\sum_{j \in N(i)} A_{i j}
$$

Now, let $i$ and $j$ both be indices of boundary points. For analyzing this case we use the notation depicted in Figure 3.

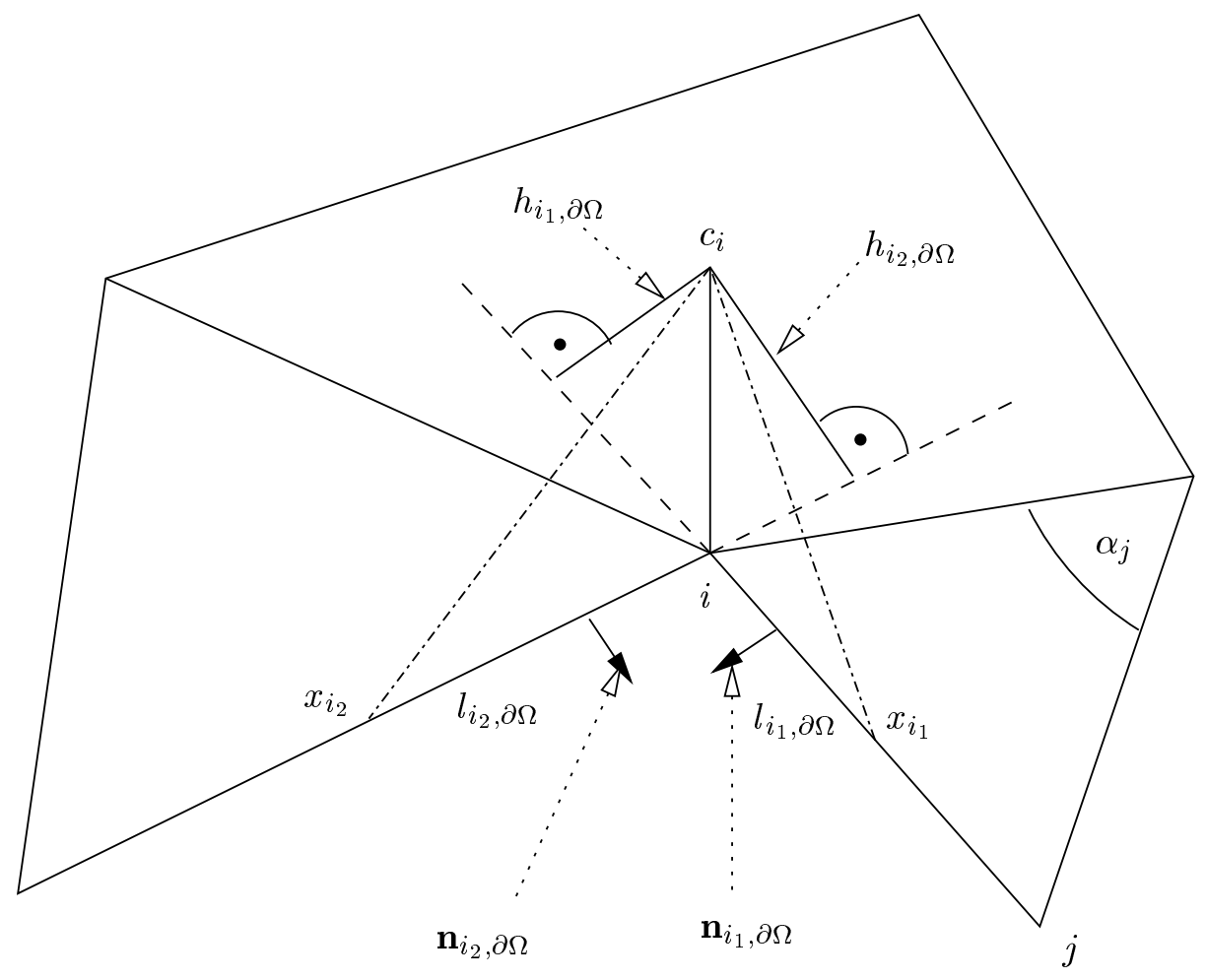

Figure 3: Sketch of the situation incorporating the extended barycenter $c_{i}$.

Then, the fluxes over the boundary edges are approximated by

$$
\int_{\ell_{i_{k}, \partial \Omega}} \lambda \nabla T \cdot \mathbf{n} d s=\lambda\left|\ell_{i_{k}, \partial \Omega}\right| \frac{T_{i}-T_{i_{k}, \partial \Omega}}{\left|h_{i_{k}, \partial \Omega}\right|}
$$

This means, that for $i \in \mathcal{N}_{h}^{\partial \mathcal{D}_{h}}$ and thus for a boundary control volume $\sigma_{i}$ the equation

$$
-\frac{\Delta t}{\left|\sigma_{i}\right|} \sum_{k=1}^{2} H\left(T_{i}, T_{i_{k}, \partial \Omega}\left(x_{i}\right), T_{i_{k}, \partial \Omega}\left(x_{i_{k}}\right) ; \mathbf{n}_{i_{k}, \partial \Omega}\right)\left|\ell_{i_{k}, \partial \Omega}\right|
$$




$$
=-\frac{\lambda \Delta t}{\left|\sigma_{i}\right|} \sum_{k=1}^{2} \frac{T_{i}-T_{i_{k}, \partial \Omega}}{\left|h_{i_{k}, \partial \Omega}\right|}\left|\ell_{i_{k}, \partial \Omega}\right|
$$

holds. Thus, by the use of the abbreviations $\mathcal{N}(i):=N(i) \cap \mathcal{N}_{h}^{\mathcal{D}_{h}}$ and $\mathcal{N}_{\partial}(i):=N(i) \cap \mathcal{N}_{h}^{\partial \mathcal{D}_{h}}$, we obtain for general $i \in \mathcal{N}_{h}$ the identity

$$
A_{i i} T_{i}+\sum_{j \in \mathcal{N}(i)} A_{i j} T_{j}+\delta_{i} \sum_{j \in \mathcal{N}_{\partial}(i)} \tilde{A}_{i j} T_{j}=\delta_{i} \sum_{k=1}^{2} A_{i, i_{k}} T_{i_{k}, \partial \Omega}
$$

where the coefficients are given as

$$
\begin{aligned}
A_{i j} \text { is defined by }(6), & \\
\tilde{A}_{i j} & =-\frac{\lambda \Delta t}{2\left|\sigma_{i}\right|} \cot \alpha_{j} \stackrel{\mathrm{A} 2}{<} 0 \quad \text { by use of }(5), \\
A_{i, i_{k}} & =\frac{\lambda \Delta t}{\left|\sigma_{i}\right|} \frac{1}{\left|h_{i_{k}, \partial \Omega}\right|}>0 \quad \text { by }(7), \\
\text { and } \quad A_{i i} & =-\sum_{j \in \mathcal{N}(i)} A_{i j}-\delta_{i} \sum_{j \in \mathcal{N}_{\partial}(i)} \tilde{A}_{i j}+\delta_{i} \sum_{k=1}^{2} A_{i, i_{k}}>0 .
\end{aligned}
$$

Thereby, the matrix $A$ described by (8) is irreducibel since any two arbitrarily chosen but fixed nodes within the computational domain are connected by our construction. Now, let a steady state solution be given with

$$
T_{i}=\max _{j \in \mathcal{N}_{h}, \ell \in \mathcal{N}_{h}^{\partial \mathcal{D}}}\left\{T_{j}, T_{\ell_{1}, \partial \Omega}, T_{\ell_{2}, \partial \Omega}\right\} .
$$

Then, by (8) we obtain for the update the condition

$$
\sum_{j \in \mathcal{N}(i)} \underbrace{A_{i j}}_{<0} \underbrace{\left(T_{j}-T_{i}\right)}_{\leq 0}+\delta_{i} \sum_{j \in \mathcal{N}_{\partial}(i)} \underbrace{\tilde{A}_{i j}}_{<0} \underbrace{\left(T_{j}-T_{i}\right)}_{\leq 0}+\delta_{i} \sum_{k=1}^{2} \underbrace{A_{i, i_{k}}}_{>0} \underbrace{\left(T_{i}-T_{i_{k}, \partial \Omega}\right)}_{\geq 0}=0 .
$$

Equality within (9) is in general obviously only guaranteed if and only if

$$
T_{i}=T_{j} \forall j \in N(i) \quad \text { and } \quad T_{i}=T_{i_{1}, \partial \Omega}=T_{i_{2}, \partial \Omega} \text { if } i \in \mathcal{N}_{h}^{\partial \mathcal{D}_{h}} .
$$

Thus, since $A$ is irreducibel it follows

$$
T_{i}=T_{j} \forall j \in \mathcal{N}_{h} \quad \text { and } \quad T_{i}=T_{i_{1}, \partial \Omega}=T_{i_{2}, \partial \Omega} \forall i \in \mathcal{N}_{h}^{\partial \mathcal{D}_{h}} .
$$

As it becomes evident within the proof, the difference between a straightforward application of the Galerkin method and our scheme if formulated as a finite element method is given by additional lines and entries within the system of equations (8) corresponding to $i \in \mathcal{N}_{h}^{\partial \mathcal{N}_{h}}$ and $j \in \mathcal{N}_{\partial}(i)$, see especially [16].

Note that the use of the concept of extended barycenters leads to more lines and entries as well as to a more natural formulation of the method in terms of physically relevant fluxes. The consideration of temperatures at the true boundary of the computational domain has no direct influence on the structure of the system (8). As noted before, it may yield advantages on the modeling side as it is the case for the industrial application we consider later. 


\section{Anisotropic adaptation}

We briefly introduce the anisotropic mesh adaptation (AMA) method elaborated in [8, 9]. This technique does not rely on the underlying problem and its exact formulation, e.g. in terms of underlying approximation techniques. Thus, it can easily be employed in the context of various types of sources, boundary conditions, etc.. For other works concerning anisotropic mesh adaptation, see e.g. [1] and the references therein.

\subsection{Theoretical background}

Let $\mathcal{D}_{h}=\left\{\mathcal{D}_{i}\right\}_{i \in I}$ ( $I=$ an index set) be a triangulation of the computational domain $\Omega$. Let $T$ be the exact solution of the continuous problem (1) and $T_{h}$ the approximate solution given by the numerical scheme (4). We recall that the numerical solution $T_{h}$ depends on $\mathcal{D}_{h}$. Now, we proceed with

Definition 1 Let $T$ and $T_{h}$ be the exact and numerical solution of the problem (1), respectively. The interpolation error function $\eta_{I}(\mathbf{x})$ is given by $($ see $[9,7])$

$$
\eta_{I}(\mathbf{x})=\left|T(\mathbf{x})-T_{h}(\mathbf{x})\right|, \mathbf{x} \in \Omega .
$$

Let $\omega>0$ be a given tolerance. Our aim is to adapt the triangulation $\mathcal{D}_{h}$ in a way that the interpolation error function satisfies the condition

$$
\eta_{I}(\mathbf{x}) \leq \omega, \quad \text { for all } \mathbf{x} \in \Omega
$$

In [9] a necessary condition on $\mathcal{D}_{h}$ is derived so that the relation (11) is fulfilled. That necessary condition depends on the exact solution $T$ and not on the approximate solution $T_{h}$. The task is now to define a triangulation satisfying the necessary condition described in [9] while the number of elements is as small as possible. Therefore, let $\mathcal{E}_{h}=\left\{e_{k}\right\}_{k \in K}$ ( $K=$ an index set) be the set of all edges of the triangulation $\mathcal{D}_{h}$. By $Q_{k}, k \in K$, we denote the center of $e_{k}$. Let us suppose that the exact solution $T$ has continuous second order derivatives with respect to the spatial variables, i.e., $T \in C^{2}(\Omega)$. Additionally, we assume that a sensible continuation of the second order derivatives to the boundary of $\Omega$ can be defined, i.e., we write $T \in C^{2}(\bar{\Omega})$. We define $\boldsymbol{H}_{k}, k \in K$, as the Hessian matrix evaluated in $Q_{k}$

$$
\boldsymbol{H}_{k}=\left.\left(\begin{array}{cc}
\frac{\partial^{2} T}{\partial x_{1}^{2}} & \frac{\partial^{2} T}{\partial x_{1} \partial x_{2}} \\
\frac{\partial^{2} T}{\partial x_{1} \partial x_{2}} & \frac{\partial^{2} T}{\partial x_{2}^{2}}
\end{array}\right)\right|_{Q_{k}}, \quad k \in K .
$$

Moreover we suppose that $\boldsymbol{H}_{k}, k \in K$, are positive definite matrices. We define the norm of the edge $e_{k}$ corresponding to $\boldsymbol{H}_{k}$ by

$$
\left\|e_{k}\right\|_{\boldsymbol{H}_{k}}:=\left(e_{k}^{\mathrm{T}} \boldsymbol{H}_{k} e_{k}\right)^{\frac{1}{2}}
$$


where $e_{k}$ is considered as a column vector in $\mathbf{R}^{2}, e_{k}^{\mathrm{T}}$ is the transposed vector and $e_{k}^{\mathrm{T}} \boldsymbol{H}_{k} e_{k}$ denotes the corresponding vector-matrix-vector product.

Now we proceed to the following

Definition 2 Let $\mathcal{D}_{h}$ be a triangulation of the computational domain $\Omega$ and $\mathcal{E}_{h}=\left\{e_{k}\right\}_{k \in K}$ be the set of all edges of $\mathcal{D}_{h}$. Let $T \in C^{2}(\bar{\Omega})$ be the exact solution of (1) and the Hessian matrices $\boldsymbol{H}_{k}, k \in K$, given by (12) are positively definite. We say that the triangulation $\mathcal{D}_{h}$ is edge-optimal, if

$$
\left\|e_{k}\right\|_{\boldsymbol{H}_{k}}=\sqrt{6 \omega} \quad \forall k \in K
$$

We now define a parameter which indicates how close the triangulation $\mathcal{D}_{h}$ is to the edgeoptimal one.

Definition 3 Let $\mathcal{D}_{h}$ be a triangulation of $\Omega$ and let $\mathbf{H}_{k}$ be a symmetric positive definite $2 \times 2$ matrix defined for each node $e_{k}, k \in K$, of $\mathcal{D}_{h}$.

Then the quality parameter of the triangulation $\mathcal{D}_{h}$ is given by

$$
Q_{\mathcal{D}_{h}}=\frac{1}{\# \mathcal{E}_{h}} \sum_{k \in K}\left(\left\|e_{k}\right\|_{\boldsymbol{H}_{k}}-\sqrt{6 \omega}\right)^{2}
$$

where $\left\|e_{k}\right\|_{\boldsymbol{H}_{k}}$ is the norm of the edge $e_{k}$ of $\mathcal{D}_{h}$ and $\# \mathcal{E}_{h}$ denotes the number of edges of $\mathcal{D}_{h}$.

It is clear that $Q_{\mathcal{D}_{h}} \geq 0$ is always satisfied and it is also clear that $Q_{\mathcal{D}_{h}}=0$ holds if the mesh $\mathcal{D}_{h}$ is edge-optimal. The aim is now to adapt a given triangulation in order to minimize the quality parameter $Q_{\mathcal{D}_{h}}$.

\subsection{Optimization of the mesh}

Of course it is practically not possible to set the matrixes $\boldsymbol{H}_{k}, k \in K$, according to (12) because we do not know the exact solution $T$. Instead of $T$ we use a smoothed version of the approximate numerical solution $T_{h}$ which we denote as $\tilde{T}_{h}$, for more detail see $[8,9]$. In general, the matrices $\boldsymbol{H}_{k}, k \in K$, are symmetric but not positive definite. Moreover, they can be singular (e.g. if $\tilde{T}_{h}$ is linear on $\Omega$ ). To overcome these difficulties, we define matrices

$$
\boldsymbol{M}_{k}=c\left[\mathbf{I}+\alpha\left(\left\|\boldsymbol{H}_{k}\right\|\right)\left|\boldsymbol{H}_{k}\right|\right], \quad k \in K,
$$

where $c>0$ is a constant and $\alpha\left(\left\|\boldsymbol{H}_{k}\right\|\right)$ is a function of the norms of the matrix $\boldsymbol{H}_{k}$. We put $\|\boldsymbol{H}\|=\max _{i, j=1,2}\left|h_{i j}\right|$, where $h_{i j}, i, j=1,2$, are the elements of $\boldsymbol{H}$. The symbol $\left|\boldsymbol{H}_{k}\right|$ denotes the absolute value of the matrix $\boldsymbol{H}_{k}$ described as follows. As $\boldsymbol{H}_{k}$ is symmetric, it can be diagonalized by

$$
\boldsymbol{H}_{k}=\mathbf{R}_{k} \operatorname{diag}\left(\lambda_{k 1}, \lambda_{k 2}\right) \mathbf{R}_{k}^{-1}, k \in K
$$


where $\lambda_{k 1}, \lambda_{k 2}$ are the eigenvalues of $\boldsymbol{H}_{k}$ and $\mathbf{R}_{k}$ is the matrix composed of the columns of eigenvectors of $\boldsymbol{H}_{k}$. We put

$$
\left|\boldsymbol{H}_{k}\right|=\mathbf{R}_{k} \operatorname{diag}\left(\left|\lambda_{k 1}\right|,\left|\lambda_{k 2}\right|\right) \mathbf{R}_{k}^{-1}, k \in K
$$

Now we define an iterative process which consists of a finite combination of the following local operations on the mesh:

- Adding a node in the center of an edge,

- Removing an edge,

- Swapping the diagonal of the quadrilateral formed by any pair of adjacent elements,

- Moving a node into a more suitable position.

These operations are performed in order to decrease the quality parameter $Q_{\mathcal{D}_{h}}$, for more details see $[8,9]$.

\section{Applications}

We consider two variations of the governing model equation (1).

First, we discuss a simple model situation. We compare some exact solutions with the corresponding results from the described numerical method, and we investigate the number of triangles needed for adaptation.

Then we consider a model of thermoregulation of premature infants. For more information on that topic, see e.g. [5, 23, 24]. In this case, our interest is not focused on the comparison with an exact solution since this is not available: instead, we investigate the number of triangles needed in order to discretize the employed two-dimensional form of the shape of an infant. We then compare the number of triangles that the adaptive scheme needs with the number of triangles used within an accurate model investigated by Fischer et al. [12] which does not feature an adaptive routine.

\subsection{Boundary layer problem with analytic solution}

The governing equation of this model problem is given by

$$
\partial_{t} T(\mathbf{x}, t)=\operatorname{div} \nabla T(\mathbf{x}, t)+y^{\prime \prime}\left(x_{1}\right)
$$

where we set $y\left(x_{1}\right)=x_{1}^{\theta}$ with $\theta \bmod 2=0$. The domain of interest is given by $[-1,1] \times$ $[-1,1] \subset \mathbf{R}^{2}$ where we employ periodic boundary conditions at the boundaries parallel to the $x_{1}$-axis and the Dirichlet condition $T=1$ at the boundaries parallel to the $x_{2}$-axis. 


\begin{tabular}{|c||c|}
\hline Mesh & Number of triangles \\
\hline \hline Original Mesh & 572 \\
First Refinement & 2040 \\
Second Refinement & 6216 \\
\hline
\end{tabular}

Table 1: Comparison of the number of triangles obtained via the refinement steps in the case $\theta=6$.

Additionally, a fixed line is set along the $x_{1}$-axis where we will measure the values of the quantity $T$ within the control volumes in order to compare these data with the values of the exact solution.

As can easily be verified, the analytical steady state solution of the described problem depends only on $x_{1}$ and is given by

$$
T\left(x_{1}\right)=2-x_{1}^{\theta}
$$

The solution features strong gradients alone in $x_{1}$-direction at the left and right boundary, while it is everywhere constant in $x_{2}$-direction. Thus, an ideal triangulation features elements near the left and right boundary showing the following properties: thin in $x_{1}$ direction and large in $x_{2}$-direction. Obviously, this is an ideal test case for an anisotropic refinement procedure.

At first, we set $\theta=6$. We start the steady state computations with a very coarse grid, see Figure 4 on page 15. To the right of that grid a comparison of the values of different numerical solutions along the $x_{1}$-axis corresponding to different steps of grid refinement is given, see Figure 5. Within the Figures 6 to 7 the grids as well as the numerical solutions resulting from two refinement steps are displayed. Since the gradients are not too strong, a good approximation of the real solution is reached by means of two adaption steps.

The comparison of the number of elements and the corresponding numerical results shows, that the use of the AMA technique results in a significant improvement of the quality of the numerical approximation while the number of triangles remains by our experiences within very reasonable bounds, see Table 4.1 .

In order to test the method in the context of thin boundary layers with steep gradients we choose the parameter $\theta=20$ and we start from an equally coarse grid as before, see Figure 8 on page 16. Again, in Figure 9 the comparison of computed values along the $x_{1}$-axis for different levels of refinement is given, while the evolution of the refinement of the grid together with the corresponding numerical solutions can be seen in the Figures 10 to 12 (pages 17 and 18).

The distribution along the $x_{1}$-axis depicted in Figure 9 as well as the coloured plots shown in the Figures 10, 11 and 12 prove that the dominant features are resolved in a reliable and accurate manner. Note that only one more level of refinement is needed to resolve a much thinner boundary layer. The anisotropic character of the refined grids is clearly observable in the transition zone between the interior and the boundary. 


\subsection{Thermoregulation}

Suddenly occuring lack of oxygen is known to be an important cause of injury to the developing brain of premature infants [18]. The neuronal loss suffered in such a critical situation evolves over several days [14]. One of the factors that influence the degree and distribution of neuronal loss is the cerebral temperature. Clinical studies have shown that lowering the cerebral temperature can prevent much damage [6]. The question arises, whether the temperature within the brain of a premature infant can be lowered by manipulating the environmental parameters in an incubator. For other works on exactly this topic, see [5] and the references therein.

The evolution of the temperature distribution within the body of a premature infant can be studied by using a variation of a so-called bio-heat equation, see e.g. [5, 23, 24]. Realistic models especially feature strong source terms by metabolical heat production and blood flow which vary corresponding to the tissue within the body. There are very strong sources due to metabolical heat production within the core, while this influence is negligible within tissue layers corresponding to skin, fat and bone especially near the body surface. Furthermore, the sources due to heat transport via blood flow have a major influence within the core where blood circulation mainly takes place, while this influence is comparatively weak within the skin and totally absent within fat and bone. Note that the mentioned tissues also feature different heat conductivity coefficients and specific heat capacities employed within our model. Of course, because of the complexity of the task, no analytical solution can be given.

The numerical simulation of such a model can result in very strong temperature gradients at the boundary of the computational domain. Of special interest is of course the temperature distribution within the head.

In order to approach this problem, Fischer et al. [12] employed a finite volume technique without an adaptive routine. This underlying model is based on the assumption of a steady state situation. Concerning the grid used within the work of Fischer et al., it should be noted that structured subgrids were used in the boundary region in order to capture steep heat gradients. For more details and for a theoretical discussion of the method see [12].

In the following, we discuss the simulation of heat transfer with respect to an infant of $1 \mathrm{~kg}$ by the use of the described adaptive scheme. It is evident to start from a coarse primary grid and to proceed by computing the corresponding steady state solution. If a steady state solution is reached, the grid is adapted by using the described AMA method and again a steady state calculation is started. We now investigate different levels of refinement together with the corresponding numerical solutions. Within the Figures 13 and 14 (pages 19 and 20) the evolution the grid undergoes is displayed, and it can be seen that a suitable solution is reached three refinement steps after starting from a quite coarse triangulation. In Table 4.2 the numbers of triangles used within the different steps of refinement are given together with the corresponding number of the mesh used by Fischer et al. which is denoted as the reference mesh. 


\begin{tabular}{|l||c|}
\hline Mesh & Number of triangles \\
\hline \hline First Mesh & 1822 \\
Second Mesh & 3673 \\
Third Mesh & 6731 \\
Fourth Mesh & 8956 \\
Reference Mesh & 37351 \\
\hline
\end{tabular}

Table 2: Numbers of triangles used within the refinement steps, compared to the grid employed by Fischer et al. [12].

\section{Concluding remarks and Outlook}

A finite volume method on unstructured grids for the simulation of heat conduction problems with steep gradients due to source terms is presented.

Realistic applications show a distribution of the heat conductivity which is piecewise constant. In this context, the maximum principle derived in [12] is improved in the sense that weaker assumptions with respect to the underlying triangulation are required. Thus, an anisotropic mesh adaptation method can be useful for the numerical solution of heat conduction problems.

The presented numerical examples show a high efficiency of the combination of the finite volume method with the anisotropic mesh adaptation technique. The numerical scheme (4) is sufficiently robust even for fast changing solutions on grids satisfying only conditions A1 and A2 admitting also thin and long triangles which are usually generated by AMA.

The numerical results from Section 4.1 show, that there is not a fundamental problem in capturing a solution with very steep gradients. Moreover, we obtain sufficiently precise solutions without large number of elements. This is also a desirable feature for $3 \mathrm{D}$ problems.

An extension of the numerical procedure to 3-D problems which covers many realistic situations is the natural next step. There is no principle problem in extending the finite volume scheme or the AMA technique in this direction. Preliminary work was already done in [25].

\section{Acknowledgement}

The research of V. Dolejší has been supported under the Grant No. 201/99/0267 and No. 201/00/D116 of the Czech Grant Agency and by the Grant MSM 113200007. The authors gratefully acknowledge these supports. M. Breuß and A. Meister would like to express their thanks to Prof. Dr. B. Fischer (Medical University Lübeck) and Dr. J. Koch (Dept. Basic Development, Drägerwerk AG, Lübeck) for their keen interest in the study. 


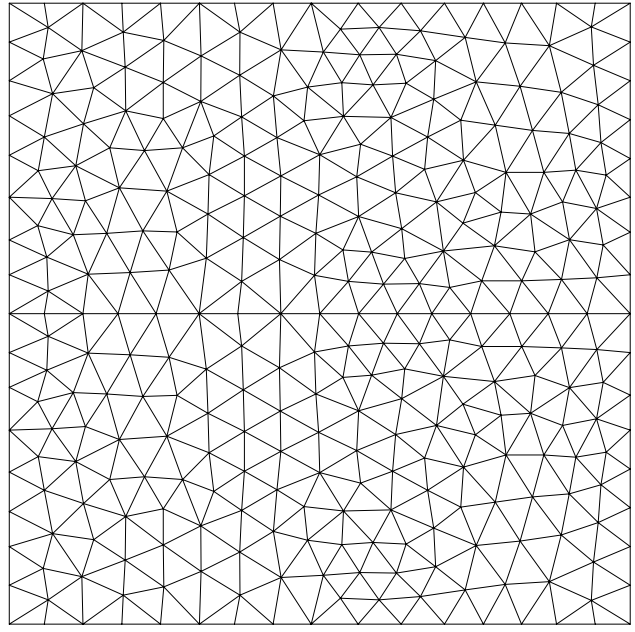

Figure 4: Initial triangulation

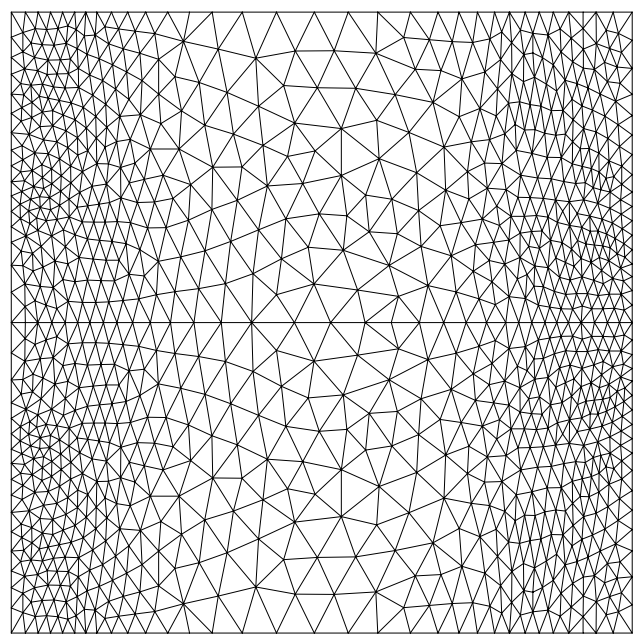

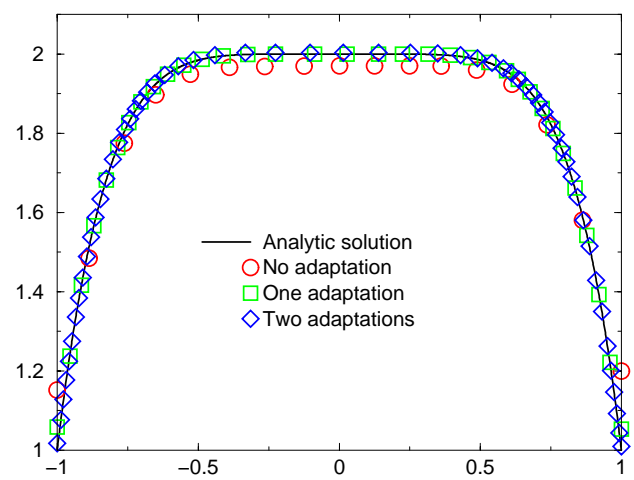

Figure 5: Temperature distribution along the $x_{1}$-axis related to the source term $y\left(x_{1}\right)=x_{1}^{6}$.

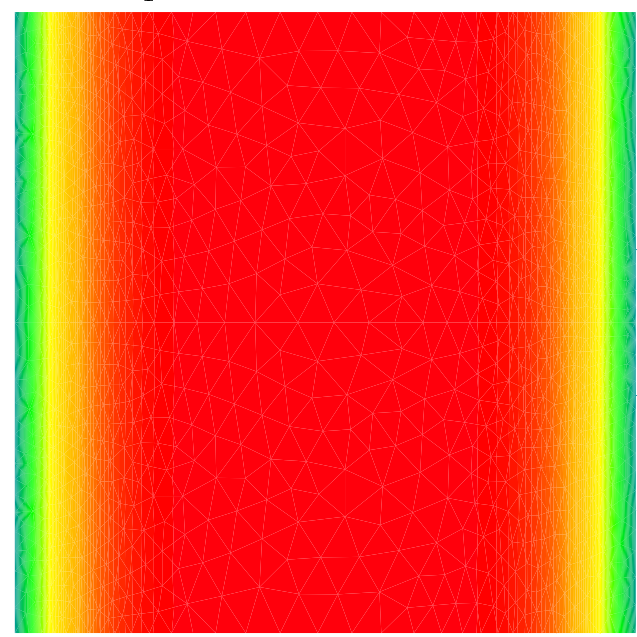

Figure 6: Grid after one adaptation and corresponding stationary temperature distribution

\section{References}

[1] Apel, T. (1999): Anisotropic finite elements: Local estimates and applications, Advances in Numerical Mathematics, Leipzig, B. G. Teubner.

[2] Bey, J. (1997): Finite Volumen und Mehrgitterverfahren für elliptische Randwertprobleme, Advances in Numerical Mathematics, Leipzig, B. G. Teubner.

[3] Breuss, M., Fischer, B. and Meister, A. (2002): The Numerical Simulation of Unsteady Heat Conduction in a Premature Infant, International Journal on Numerical Methods in Fluids 40, 253-261.

[4] Brück, K. (1961): Temperature regulation in the newborn infant, Biol. Neonate 3, $65-119$. 

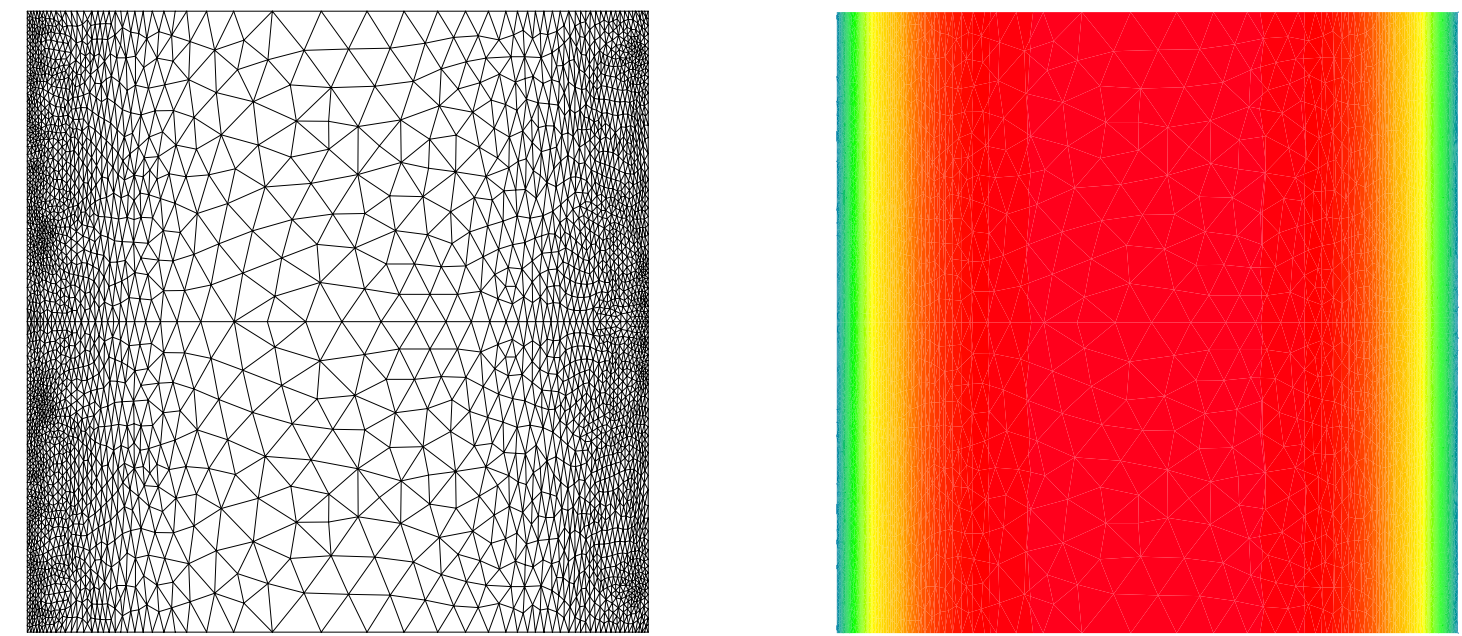

Figure 7: Grid after two adaptations and corresponding stationary temperature distribution

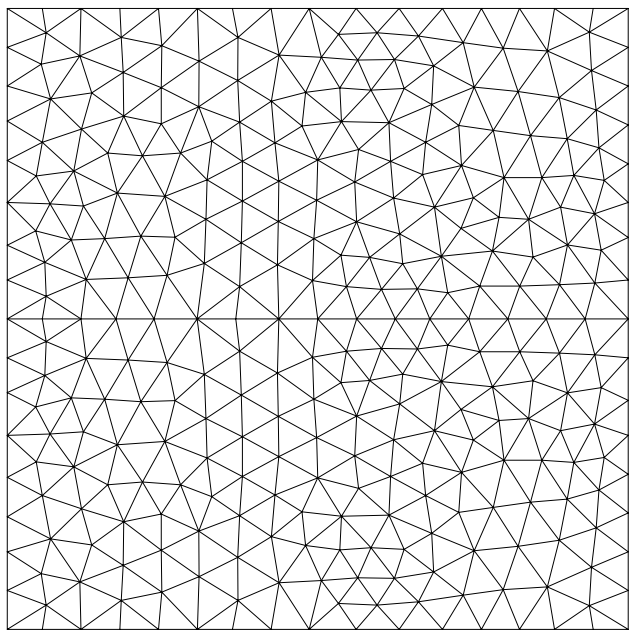

Figure 8: Initial triangulation

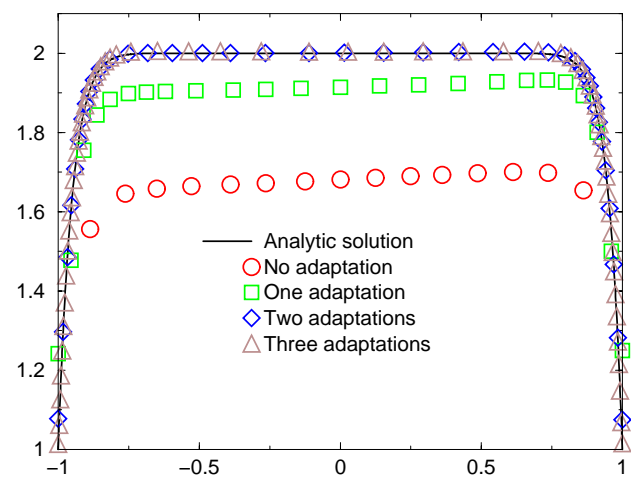

Figure 9: Temperature distribution along the $x_{1}$-axis related to the source term $y\left(x_{1}\right)=x_{1}^{20}$.

[5] Bussmann, O. (2000): Modell der Thermoregulation des Früh- und Neugeborenen unter Einbeziehung der thermischen Reife, PhD thesis, Institute of Biomedical Engineering, Medical University of Lübeck.

[6] Busto, R., Dietrich, W. D., Globus, M. Y. and Ginsberg, M.D. (1989): The importance of brain temperature in cerebral ischemic injury, Stroke 20, 1134-1114.

[7] D'Azevedo, E. F. and Simpson, R. B. (1991): On optimal triangular meshes for minimizing the gradient error, Numer. Math. 59, 321-348.

[8] Dolejší, V. (1998): Anisotropic mesh adaptation for finite volume and finite element methods on triangular meshes, Computing and Visualisation in Science 1, 165-178.

[9] Dolejší, V. and Felcman, J. (2004): Anisotropic mesh adaptation and its application for scalar diffusion equations, Numerical Methods for Partial Differential Equations 20, $576-608$. 

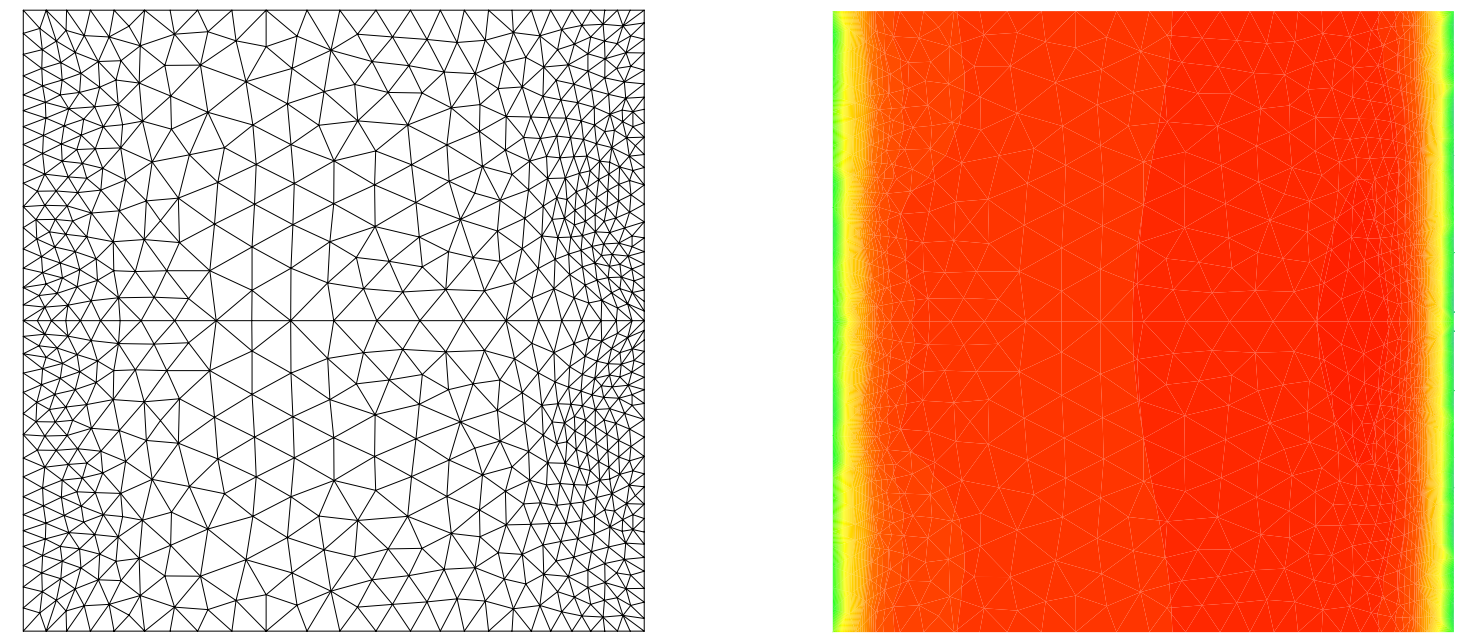

Figure 10: Grid after one adaptation and corresponding stationary temperature distribution
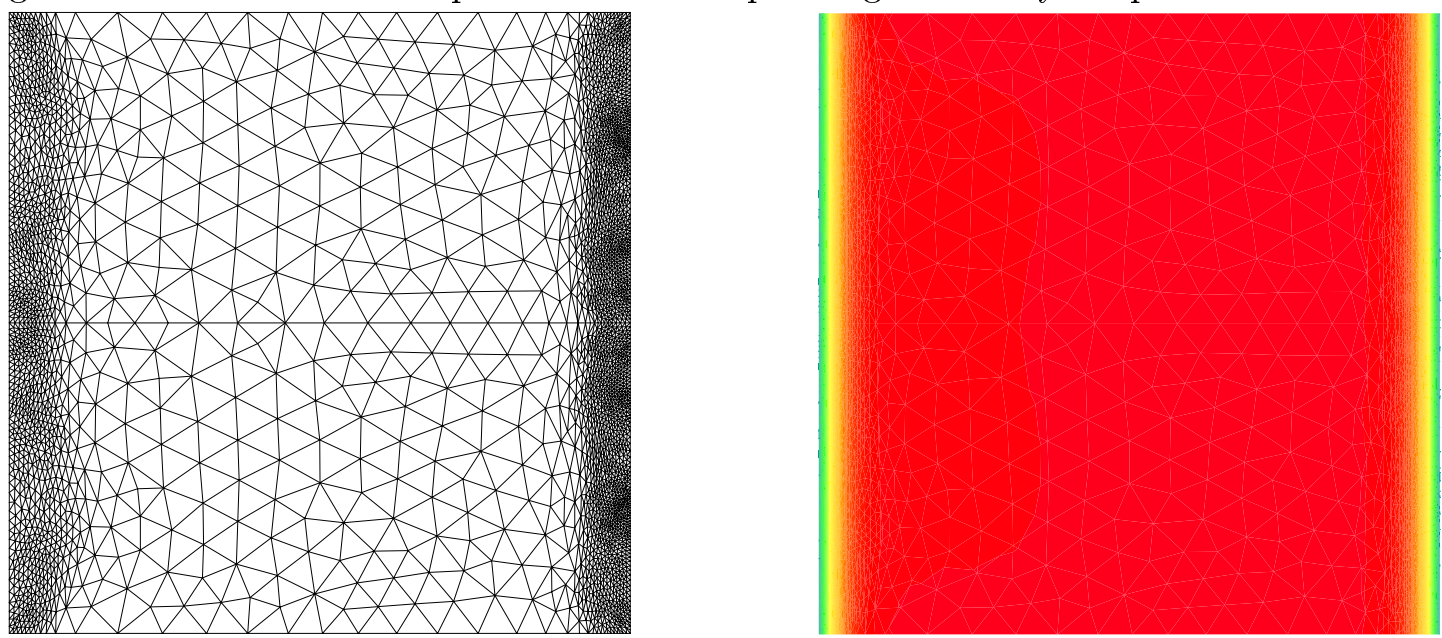

Figure 11: Grid after two adaptations and corresponding stationary temperature distribution

[10] Feistauer, M., Felcman, J. and Dolejší, V. (1996): Numerical Simulation of Compresssible Viscous flow through Cascades of Profiles, ZAMM 76, 297-300.

[11] Feistauer, M., Felcman, J. and Lukáčová, M. (1995): Combined finite elements-finite volume solution of compressible flow, J. Comput. Appl. Math. 63, 179-199

[12] Fischer, B., Ludwig, M. and Meister, A. (2001): The Thermoregulation of Infants: Modeling and Numerical Simulation, BIT 41, No. 5, 950-966.

[13] Friedrich, O., Hempel, D., Meister, A. and Sonar, Th. (1995): Adaptive Computation of Unsteady Flow Fields with the DLR- $\tau$-Code, in: 77th AGARD Fluid Dynamics Panel Meeting and Symposium on Progress and Challenges in CFD Methods and Algorithms, AGARD-CP-578, Sevilla.

[14] Gluckman, P. D. and Williams, C. E. (1992): When and why do brain cells die?, Dev. Med. Child Neurol. 34, 1010-1014. 

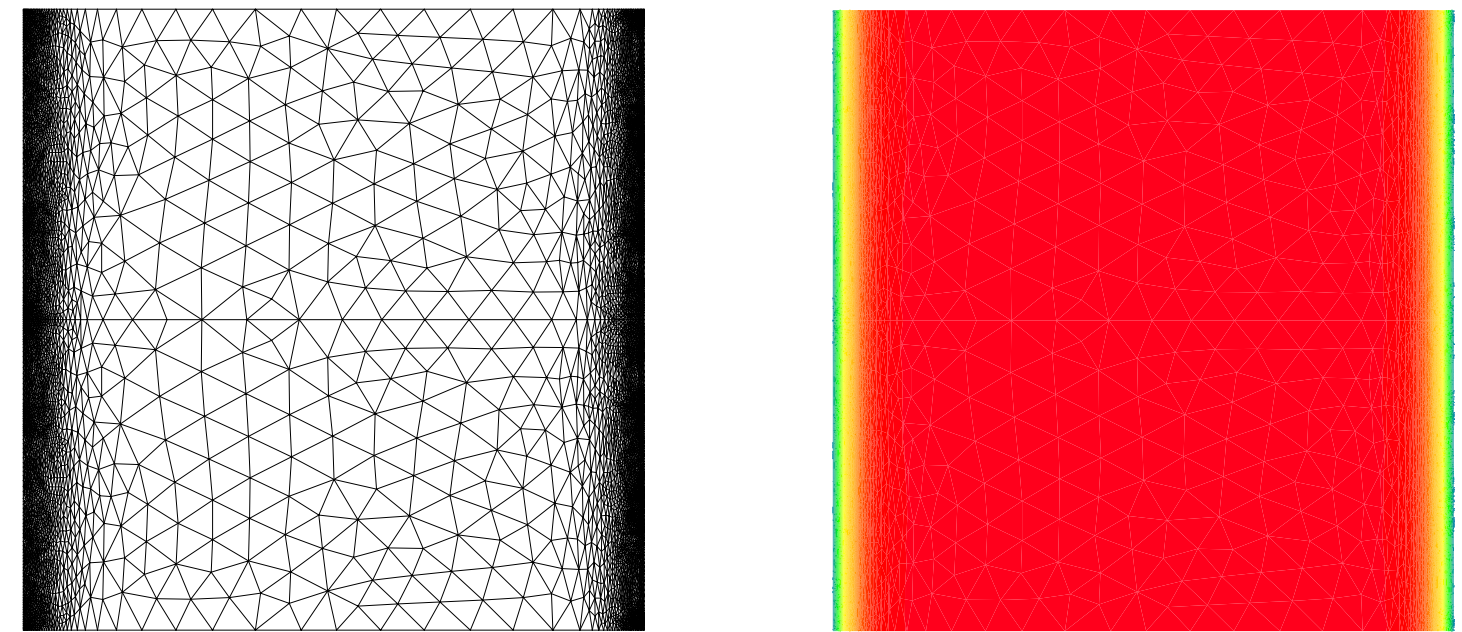

Figure 12: Grid after three adaptations and corresponding stationary temperature distribution

[15] Gray, D. D. and Giorgini, A. (1976): The validity of the Boussinesq approximation for liquids and gases, Int. J. Heat Mass Transfer 19, 545-551.

[16] Hackbusch, W. (1989): On first and second order box schemes, Computing 41, 277296.

[17] Kröner, D. (1997): Numerical Schemes for Conservation Laws, Teubner, Stuttgart.

[18] Mallard, E. C., Williams, C. E., Johnston, B. M. and Gluckman, P. D. (1995): Neuronal damage in the developing brain following intrauterine asphyxia, Reprod. Fertil. Dev. 7, 647-653.

[19] Meister, A. and Oevermann, M. (1998): An Implicit Finite Volume Approach of the $k-\epsilon$ Turbulence Model on Unstructured Grids, Z. Angew. Math. Mech. 78, No. 11, $743-757$.

[20] Meister, A. and Sonar, Th. (1998): Finite-volume schemes for compressible fluid flow, Surv. Math. Ind. 8, 1-36.

[21] Sonar, Th. (1997): Mehrdimensionale ENO-Verfahren, Teubner, Stuttgart.

[22] Sonar, Th. (1997): On the Construction of Essentially Non-Oscillatory Finite Volume Approximations to Hyperbolic Conservation Laws on General Trinagulations: Polynomial Recovery, Accuracy, and Stencil Selection, Comp. Meth. Appl. Mech. Eng. 140, $157-181$.

[23] Werner, J. (1989): Thermoregulatory models: recent research, current applications and future development, Scand. J. Work. Environ. Health 15, 34-46.

[24] Werner, J. and Webb, P. (1993): A Six-cylinder Model of Human Thermoregulation for general Use on Personal Computers, Ann. Physiol. Anthrop. 12, No. 3, 123-134.

[25] Zoubek, M. (2001): Adaptive methods for the solution of threedimensional flow, Master thesis, Charles University Prague, in Czech. 

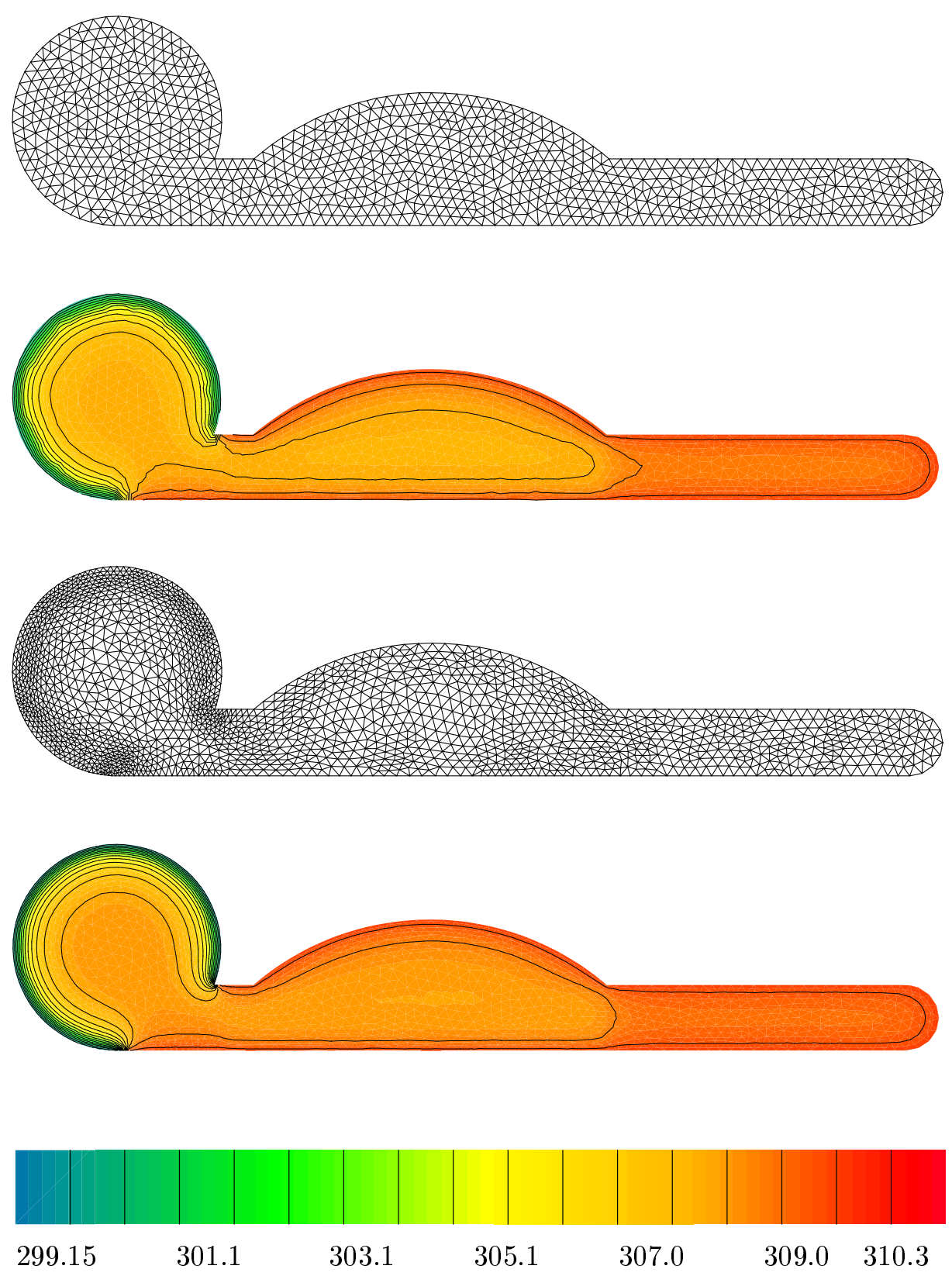

Temperature measure in $[\mathrm{K}]$

Figure 13: Sequence of triangulations and corresponding temperature distributions (part I) 

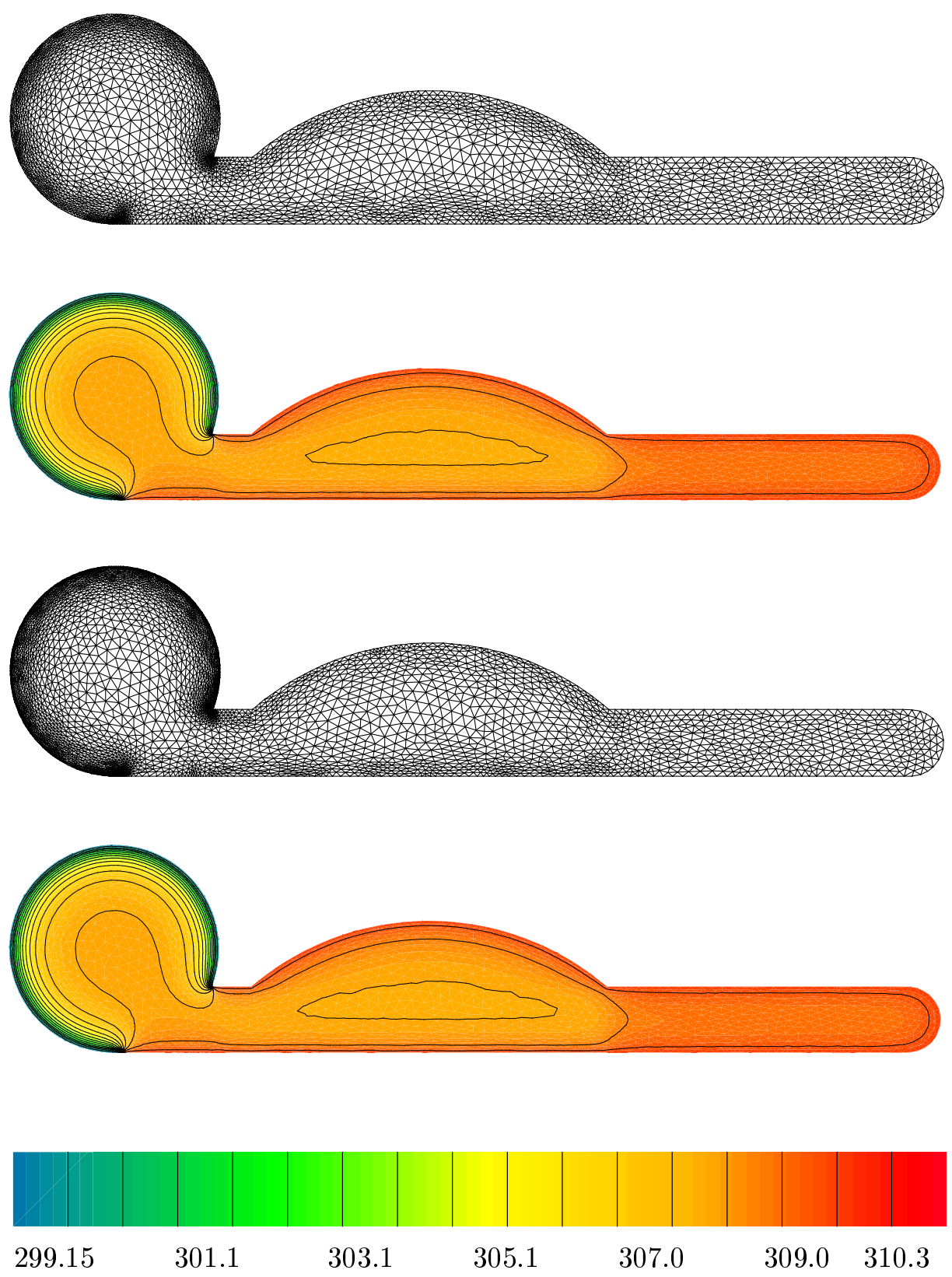

Temperature measure in $[\mathrm{K}]$

Figure 14: Sequence of triangulations and corresponding temperature distributions (part II) 